\title{
Complementing Warm-up with Stretching Routines: Effects in Sprint Performance
}

\section{두(ㅇ)(우}

\author{
Authors \\ Daniel Almeida Marinho ${ }^{1,2}$, Maria Helena Gil1,2, Mario Cardoso Marques ${ }^{1,2}$, Tiago Miguel Barbosa ${ }^{2,3}$, Henrique Pereira Neiva ${ }^{1,2}$
}

\section{Affiliations}

1 University of Beira Interior, Covilhã, Portugal, Department of Sport Sciences, Covilha, Portugal

2 Reserch Centre in Sports, Health and Human Development, CIDESD, Portugal

3 National Institute of Education, Nanyang Technological University, Physical Education \& Sports Science Academic Group, Singapore, Singapore

Key words

pre-exercise, repeated-sprint, evaluation, velocity, heart rate, tympanic temperature

received 06.03 .2017

revised 08.05 .2017

accepted 11.05 .2017

\author{
Bibliography \\ ISSN 2367-1890 \\ Correspondence \\ Dr. Daniel Almeida Marinho \\ UBI \\ Sport Sciences \\ Rua Marquês d'Ávila e Bolama \\ 6201-001, Covilhã \\ Portugal
}

DOI https://doi.org/10.1055/s-0043-111788

Sports Medicine International Open 2017; 1: E101-E106

(c) Georg Thieme Verlag KG Stuttgart · New York
Tel.: 00351275319700 , Fax: 00351275320695

marinho.d@gmail.com

\begin{abstract}
ABSTACT
The present study aimed to examine the effects of using static or dynamic stretching added to the common warm-up routine for short sprint distances and to repeated sprint performance. In 3 different sessions, 16 college-age men $(n=10)$ and women $(n=6)$ performed one of 3 warm-ups followed by a $2 \times 60 \mathrm{~m}$ dash sprint time trial ( 5 min of rest) in a counterbalanced design. The control warm-up consisted of $10 \mathrm{~min}$ of light-intensity running, and the 2 experimental warm-ups included a static or dynamic stretching routine (5 exercises) in the control warm-up. Performance (time) and physiological variables (tympanic temperature, heart rate) were monitored. In the first $60 \mathrm{~m}$ time trial, there were no differences between the 3 warmups tested $\left(F=0.21, p=0.73 ; \eta_{p}{ }^{2}=0.01\right)$, as opposed to that observed in the second $\left(F=7.04, p<0.01 ; \eta_{p}{ }^{2}=0.32\right)$. The participants were $1.7 \%$ faster after the static stretching warm-up compared with the control warm-up. The sum of the time performed in the 2 sprints emphasizes these results, with better performances after the static stretching warm-up than the control ( $1 \%)$ or dynamic stretching warm-up $(0.7 \%)$. These results suggest that including a set of static or dynamic stretching exercises may enhance sprinting performance. The better performance in the second trial after the warm-up including static stretching suggests that this type of stretching may positively influence repeated sprint performance ( $<10$ s sprint).
\end{abstract}

\section{Introduction}

Stretching during the warm-up is a key routine in training and physical fitness programmes $[14,21]$. Practitioners claim that stretching can enhance performance and reduce the incidence of musculoskeletal injury and the onset of the delayed muscle sourness $[14,29]$. However, evidence has challenged these arguments $[3,10]$.

Some researchers noted that static stretching routines caused impairments in strength, power, maximal strength development, vertical jump and short sprinting performances [10,24]. The results seems particular harmful when stretching to the point of dis- comfort, considered to be maximal stretching intensity [3, 31]. Some evidence in the literature suggests that submaximal stretching intensities (for example, $90 \%$ of maximal range of motion), might not produce such impairments [32]. However, there is more agreement that dynamic stretching seemed to produce better performance in subsequent physical bouts, even in short-duration efforts [3, 24].

Nevertheless, several methodological limitations could be noted. Most studies implemented static protocols ranging between 90 s to 30 min duration for each muscle group, which is clearly different from what usually occurs in real settings $[2,3,10]$. In field 
- Table 1 Characterization of the male and female participants.

\begin{tabular}{|l|c|c|c|c|c|}
\hline & Age (years) & Height $(\mathbf{m})$ & Body mass $(\mathbf{k g})$ & Body mass index $\left(\mathbf{k g} / \mathbf{m}^{2}\right)$ & Training experience $($ years) \\
\hline Males $(\mathrm{n}=10)$ & $22.10 \pm 1.52$ & $1.76 \pm 0.06$ & $74.23 \pm 8.57$ & $23.92 \pm 2.27$ & $2.50 \pm 1.72$ \\
\hline Females $(\mathrm{n}=6)$ & $21.83 \pm 1.72$ & $1.65 \pm 0.05$ & $54.57 \pm 4.92$ & $20.13 \pm 0.93$ & $2.83 \pm 0.75$ \\
\hline Total $(\mathrm{n}=16)$ & $22.00 \pm 1.55$ & $1.72 \pm 0.08$ & $66.86 \pm 12.20$ & $22.50 \pm 2.64$ & $2.63 \pm 1.41$ \\
\hline
\end{tabular}

settings, subjects stretch each muscle group from 10-30 s, with 2 or 3 repetitions, and mostly to the point of discomfort. Additionally, those studies tended to analyse the effect of stretching in an isolated form and not as a complementary routine to a warm-up session that aimed to increase subject preparedness for exercitation [15]. Moreover, researchers focused mostly on the evaluation of a single maximal effort and little is known about the effect of stretching on repeated maximal efforts [15].

The controversy still exists and the effect of static or dynamic stretching in maximal efforts or in repeated maximal efforts is unknown. Moreover, it seems appropriate that in activities requiring a high range of motion, the athletes should select drills preparing themselves to reach the optimal range of motion and therefore enhance performance. Accurate studies are lacking on whether adding stretching routines to the warm-up could enhance performance without any impairment [16]. Therefore, the purpose of this study was to verify the effects of added stretching (static vs. dynamic) exercises in a warm-up routine on sprinting performance and physiological response. In addition, we intended to understand the effect of both warm-ups in a second time-trial repetition. It was hypothesised that a warm-up including stretching dynamic exercitation would improve sprint performance, also leading to an increased tympanic temperature and lower heart rate responses to exercise.

\section{Material and Methods}

\section{Subjects}

A convenience sample of 16 college students ( 10 males and 6 females; $22.00 \pm 1.55$ years old; height $1.72 \pm 0.08 \mathrm{~m}$; body mass $66.86 \pm 12.20 \mathrm{~kg}$ ) took part in this study. All participants were physically active and competed at the university level for the last $2.63 \pm 1.41$ years. $>$ Table 1 presents the subjects' characteristics. After approval by the university ethics committee to ensure compliance with the Helsinki declaration, participants were informed about the study procedures and written informed consent was obtained. Additionally, this study was performed in accordance with the ethical standards proposed by Harris and Atkinson [8].

\section{Testing procedure}

The experiments were performed over a 3-week period on an official running track at the same time of the day and with similar weather conditions. Air temperature remained between $19^{\circ} \mathrm{C}$ and $21^{\circ} \mathrm{C}\left(19.80 \pm 0.92^{\circ} \mathrm{C}\right)$ and wind $<2 \mathrm{~m} / \mathrm{s}$.

The study followed a randomized crossover design. 3 warm-up procedures were tested: (i) control warm-up (no stretching routine included), (ii) static stretching warm-up (SS), and (iii) dynamic stretching warm-up (DS). Each warm-up condition was tested with
$48 \mathrm{~h}$ between them in randomized order. The subjects were familiarised with the warm-up procedures one week before the first evaluation and they were reminded to maintain the same routines during the $48 \mathrm{~h}$ prior to testing. After finishing the warm-up, each subject remained seated for $5 \mathrm{~min}$ and then completed 2 time trials of $60 \mathrm{~m}$ running ( 5 min rest between bouts).

The stretching activities were those that the athletes normally used in their daily warm-up routines. The control warm-up consisted of 10 min of continuous running at moderate intensity (50 to $70 \%$ of predicted maximal heart rate), as suggested in the literature [30]. The SS consisted of the same running activity as the control warm-up followed by a static stretching sequence of 5 exercises, completed in 8-10 min. All stretches were repeated for 3 sets of $30 \mathrm{~s}$ ( $15 \mathrm{~s}$ interval) and held at the point of mild discomfort. The static exercises included: i) hamstring stretch (grab the knee and pull it straight up, towards the chest); ii) standing quadriceps stretch (grab the foot and pull it back to gluteus); iii) standing hamstring stretch (one leg on an elevated support, bend from the lower back and reach forward, keeping the legs straight); iv) seated hamstring stretch (with the knee of one leg bent and the other leg extended, bend the waist toward the extended leg); v) lying quadriceps stretch (lie on side and pull heel toward buttocks until a stretch is felt in front of the thigh). The DS was similar but replaced static stretching with a dynamic stretching sequence of 5 exercises, completed in 8 to $10 \mathrm{~min}$. The dynamic stretches were performed over a $20 \mathrm{~m}$ course and the exercises used were the same as those of Turki et al. [27].

Official start commands were used and time trials started from the official starting block. The $60 \mathrm{~m}$ trial was chosen because it is the shortest IAAF event. In addition, research with regard to the influence of warm-up at this particular distance and in repeated $60 \mathrm{~m}$ sprints is scarce [15]. Time trial performances were recorded by photocells (Polifemo Radio, Microgate, Bolzano-Bozen, Italy) at the $0,20,40$, and $60 \mathrm{~m}$ mark and at $1.17 \mathrm{~m}$ above the floor.

After arriving at the track, the athletes remained seated for $5 \mathrm{~min}$, with the legs uncrossed, to assess baseline measurements. Tympanic temperature measurements were assessed before the warm-up (baseline measures), immediately before each one of the two $60 \mathrm{~m}$ bouts ( $1 \mathrm{~min}$ ), and $5 \mathrm{~min}$ into recovery. This is a good indicator of brain temperature, which controls body temperature, and each tympanic temperature was taken 3 times, and the maximal value was recorded (Braun Thermoscan IRT 4520, Germany). The thermometers had a measuring accuracy of $0.2^{\circ} \mathrm{C}$ for temperatures between 32.0 and $42.0^{\circ} \mathrm{C}$. The heart rate was also assessed at baseline, immediately before each trial ( $1 \mathrm{~min}$ ) and $5 \mathrm{~min}$ after the second $60 \mathrm{~m}$ bout (Vantage NV; Polar, Lempele, Finland). During that time, the participants remained seated. Each physiological measurement was performed 3 times, for each evaluation, and the highest value was recorded (ICC $>0.97$ ). 

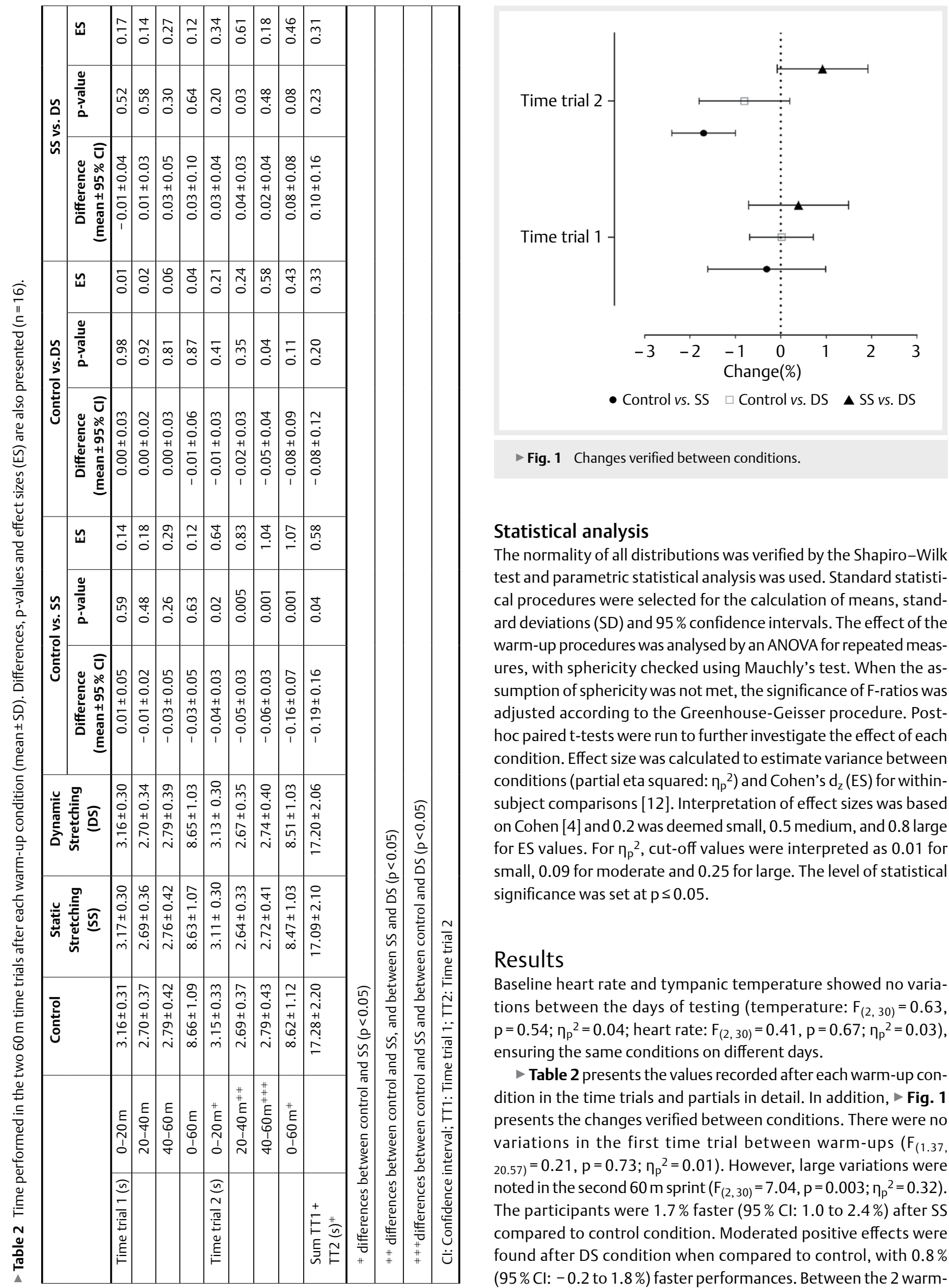

\section{Statistical analysis}

The normality of all distributions was verified by the Shapiro-Wilk test and parametric statistical analysis was used. Standard statistical procedures were selected for the calculation of means, standard deviations (SD) and $95 \%$ confidence intervals. The effect of the warm-up procedures was analysed by an ANOVA for repeated measures, with sphericity checked using Mauchly's test. When the assumption of sphericity was not met, the significance of F-ratios was adjusted according to the Greenhouse-Geisser procedure. Posthoc paired t-tests were run to further investigate the effect of each condition. Effect size was calculated to estimate variance between conditions (partial eta squared: $\eta_{p}{ }^{2}$ ) and Cohen's $d_{z}(E S)$ for withinsubject comparisons [12]. Interpretation of effect sizes was based on Cohen [4] and 0.2 was deemed small, 0.5 medium, and 0.8 large for $E S$ values. For $\eta_{p}^{2}$, cut-off values were interpreted as 0.01 for small, 0.09 for moderate and 0.25 for large. The level of statistical significance was set at $\mathrm{p} \leq 0.05$.

\section{Results}

Baseline heart rate and tympanic temperature showed no variations between the days of testing (temperature: $F_{(2,30)}=0.63$, $p=0.54 ; \eta_{p}^{2}=0.04$; heart rate: $\left.F_{(2,30)}=0.41, p=0.67 ; \eta_{p}^{2}=0.03\right)$, ensuring the same conditions on different days.

- Table 2 presents the values recorded after each warm-up condition in the time trials and partials in detail. In addition, > Fig. 1 presents the changes verified between conditions. There were no variations in the first time trial between warm-ups $\left(F_{(1.37}\right.$, $\left.20.57)=0.21, p=0.73 ; \eta_{p}{ }^{2}=0.01\right)$. However, large variations were noted in the second $60 \mathrm{~m}$ sprint $\left(\mathrm{F}_{(2,30)}=7.04, \mathrm{p}=0.003 ; \eta_{\mathrm{p}}{ }^{2}=0.32\right)$. The participants were $1.7 \%$ faster ( $95 \% \mathrm{Cl}: 1.0$ to $2.4 \%$ ) after SS compared to control condition. Moderated positive effects were found after DS condition when compared to control, with $0.8 \%$ ( $95 \% \mathrm{Cl}:-0.2$ to $1.8 \%$ ) faster performances. Between the 2 warm- 


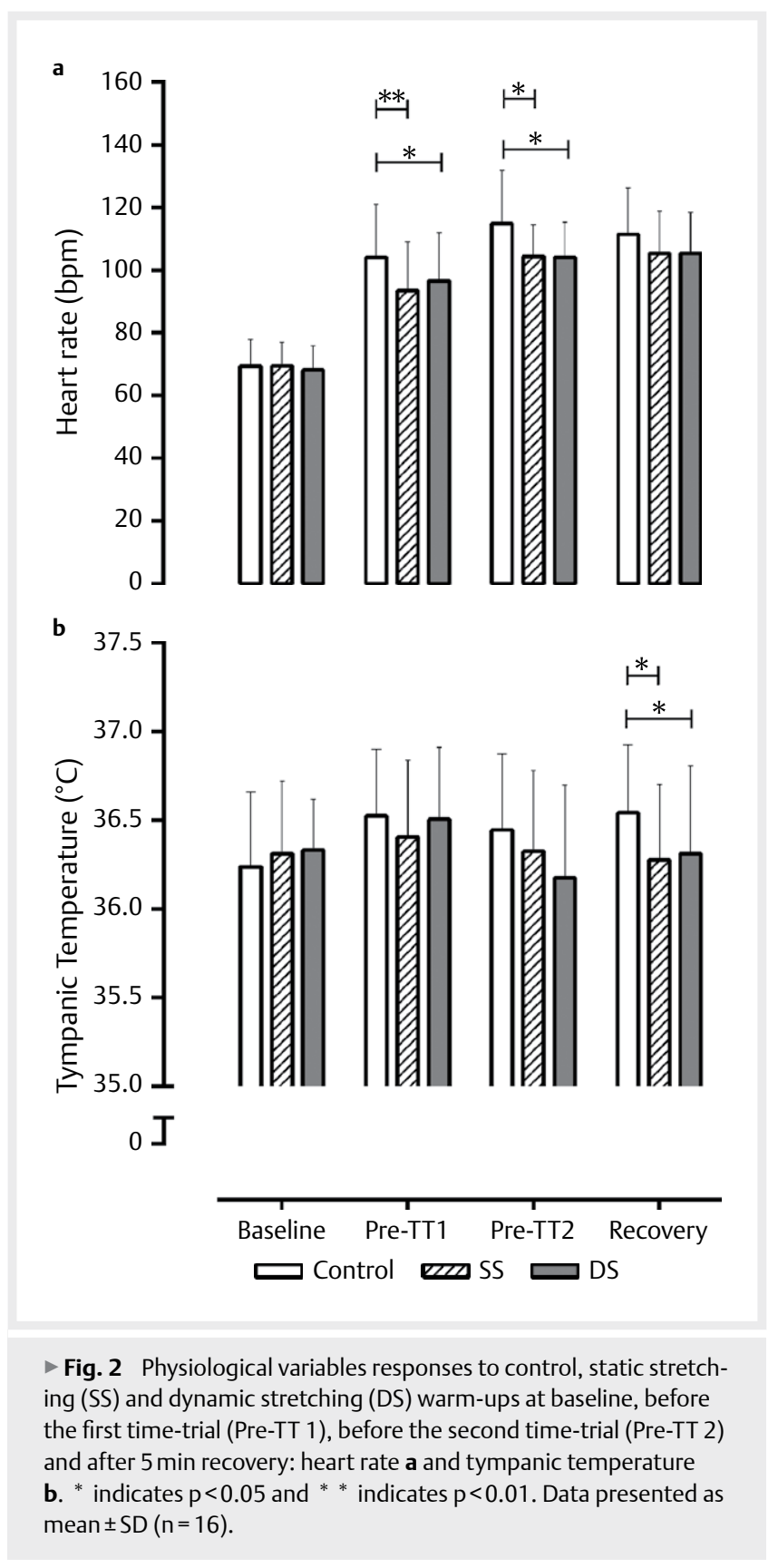

ups that included stretching routines, the SS produced better performances $(0.9 \%$; $95 \% \mathrm{Cl}:-0.1$ to $1.8 \%)$.

The values obtained from the sum of the 2 time trials highlighted the benefit of SS, with lower times than either the control warmup ( $1.0 \%$; $95 \% \mathrm{Cl}: 0.2$ to $1.9 \%)$ or DS $(0.6 \% ; 95 \% \mathrm{Cl}:-0.3$ to $1.5 \%)$.

As far as the physiological variables are concerned, the heart rate was different between conditions before the first sprint $\left(\mathrm{F}_{(2,28)}=5.10\right.$, $\left.p=0.01 ; \eta_{p}{ }^{2}=0.27\right)$ and the second sprint $\left(F_{(1.28,17.98)}=4.017\right.$, $\left.p=0.05 ; \eta_{p}^{2}=0.22\right)$. Higher values were found in the control condition compared to SS (first sprint: $p=0.01$, ES $=0.82$; second sprint: $p=0.02, E S=0.70$, or DS (first sprint: $p=0.04, E S=0.67$; second sprint: $p=0.05, E S=0.55)$. No differences were found in tympanic temperatures before the first $\left(F_{(2,30)}=0.86, p=0.43 ; \eta_{p}{ }^{2}=0.05\right)$ and the second trial $\left(F_{(2,30)}=2.59, p=0.09 ; \eta_{p}{ }^{2}=0.15\right)$. Significance ap- peared only after the 5 min of recovery $\left(F_{(2,30)}=3.32, p=0.05\right.$; $\left.\eta_{\mathrm{p}}{ }^{2}=0.18\right)$, with increased values of the control condition compared to $S S(p=0.02$, ES $=0.64)$ or DS $(p=0.04, E S=0.55)$. - Fig. 2 illustrates these results with respect to the physiological variables.

\section{Discussion}

The main aim of this study was to compare the effects of stretching during a warm-up routine before a short-distance sprint $(60 \mathrm{~m})$. In addition, it was intended to verify the influence of different warm-ups in repeated-sprint performance. There were no differences between conditions in the first $60 \mathrm{~m}$ sprint, suggesting that including static or dynamic stretching after a light-intensity continuous run does not affect sprinting performance. However, a second repetition of $60 \mathrm{~m}$ improved when stretching was included in the warm-up routine, notably static stretching.

The different warm-ups evaluated did not show differences in the first time-trial performance. These results are contrary to other studies reporting the benefits of dynamic instead of static stretching $[3,6,20]$. The dynamic stretches could improve the performance in the short sprint and all-out bouts because of the similarity of motor pattern used, the increased body temperature obtained by the movement, the proprioceptive facilitation and better pre-activation for the subsequent task $[6,20]$. On the other hand, static stretching is expected to affect musculoskeletal stiffness, leading to an impairment of the potential elastic energy stored by the stretch-shortening cycle [28], and/or to more challenging neuromuscular stimulation because of the diminished activity by the muscle proprioceptors [11]. Nevertheless, there were reports that muscle-tendon properties "in vivo" remained unchanged after the static stretch, which is not in line with the evidence reported early on [19]. Moreover, Kay and Blazevich [10] mentioned that most studies on this topic did not observe impaired performances in strength, power, or velocity when stretching for less than $45 \mathrm{~s}$. Negative effects arose only when static stretching was performed for more than $60 \mathrm{~s}$. The results of the present study partially support this report.

In the SS warm-up, participants were better in each $20 \mathrm{~m}$ split and hence in the $60 \mathrm{~m}$ time trial. The role of the first maximal repetition seemed to be a key factor for these results. Performing dynamic movements and activities after static stretching could reduce the possible negative effect on performance, reversing any undesirable muscular effect or associated neural effects $[13,25]$. Furthermore, recent evidence showed that only 10 min should be needed to restore the maximal values of isometric strength after 5 min of static stretching [18]. Thus, in the present study, the second bout was held beyond this 10 min interval. Subjects could even have benefited from gains in muscular range of motion that might remain elevated for 30 min after static stretches [17]. It is plausible that one can propose a possible potentiation effect caused by the first maximal repetition. This caused an improvement in all conditions tested. This maximal activity could result in increased neuromotor excitability, which leads to a considerable increase in the rate of force development and power production [26]. Therefore, including a short-duration task at maximal intensity or even a racepace task before the race or the training main set could maximize performance. 
The acute response to warm-up showed that all 3 warm-ups elevated body temperature and heart rate, as expected [15, 22, 23]. Most of the effects of warm-up are related to an increase in body temperature, oxygen uptake and heart rate [15]. Those gains theoretically also support a positive effect on sprint performance. For instance, it is known that an increase in muscle temperature can lead to better sprint performance by increasing muscle glycogen availability in short-term efforts [7]. The temperature responses together with heart rates would allow us to interpret the performance results obtained, caused by the different warm-up conditions. In fact, heart rate is easy to monitor in the field context and shows a very stable pattern that allows coaches and athletes to verify and adjust the exercise intensity. Because the intensity of trials was maximal and the sprints were short in the present study, the acute responses were expected to be minimal. However, the heart rate adaptation to each warm-up condition during all procedures allowed us to verify different energy expenditures [9] and to explain possible causes for different performances.

Exercise intensity is usually related to the amount of energy expended to perform a certain activity [9]. In non-laboratory settings, heart rate can be used to compare energy expenditures between exercises [1]. We noted that the increase in heart rate was higher in the control warm-up, possibly resulting from the continuous activity that was completed without the "interval" associated with the stretching exercises. In fact, one possible explanation for the better performance obtained in the sum of the two $60 \mathrm{~m}$ sprints in SS could be related to the higher energetic expenditure in the other 2 warm-ups. The 5 min interval between warm-ups and the beginning of the time trials could not allow full replenishment of ATP-PCr reserves, essential to an effort of less than $10 \mathrm{~s}$ duration [5]. Contrary to the control and DS warm-up, which comprised physical effort during all warm-up protocols, the SS comprised lighter activities, very close to a resting situation. Therefore, in this warm-up condition, the energy expenditure could be almost null and could allow starting the recovery phase after the first sprint earlier than the others. This temporal gain allowed the full recovery of the energy storage and of the neuromuscular system [15].

We could also suggest that the post-activation potentiation caused by the first sprint allowed greater improvement in the second sprint after a warm-up with static stretching routines. Coaches should be aware of this evidence, not only for when athletes are competing more than once in the same competition session or during training sessions where maximal repetitions appear essential to increase preparedness, but also in intermittent sports (e.g., team sports) performance.

\section{Conclusions}

The current results suggest that including a stretching exercise routine, static or dynamic, during warm-up could be a reliable option when preparing for short- distance repeated-running performances. It was verified that the second $60 \mathrm{~m}$ repetition was faster than the first when static stretching was used as a complement to a simple running warm-up. This fact seems to suggest that the warmup, when complemented with stretching exercises, positively influences repeated sprint ability.

\section{Acknowledgements}

This project was supported by the National Funds through FCT Portuguese Foundation for Science and Technology (UID/ DTP/04045/2013) - and the European Fund for Regional Development (FEDER) allocated by European Union through the COMPETE 2020 Programme (POCI-01-0145-FEDER-006969) - competitiveness and internationalization $(\mathrm{POCl})$.

\section{Conflict of Interest}

The authors declare that they have no conflict of interest.

\section{References}

[1] Achten J, Jeukendrup AE. Heart rate monitoring: Applications and limitations. Sports Med 2003; 33: 517-538

[2] Beckett JR, Schneiker KT, Wallman KE, Dawson BT, Guelfi KJ. Effects of static stretching on repeated sprint and change of direction performance. Med Sci Sports Exerc 2009; 41: 444-450

[3] Behm DG, Chaouachi A. A review of the acute effects of static and dynamic stretching on performance. Eur J Appl Physiol 2011; 111: 2633-2651

[4] Cohen J. A power primer. Psychol Bull 1992; 112: 155-159

[5] Gastin PB. Energy system interaction and relative contribution during maximal exercise. Sports Med 2001; 31: 725-741

[6] Gelen E. Acute effects of different warm-up methods on sprint, slalom dribbling, and penalty kick performance in soccer players. J Strength Cond Res 2010; 24: 950-956

[7] Gray SR, De Vito G, Nimmo MA, Farina D, Ferguson RA. Skeletal muscle ATP turnover and muscle fiber conduction velocity are elevated at higher muscle temperatures during maximal power output development in humans. Am J Physiol Regul Integr Comp Physiol 2006; 290: R376-R382

[8] Harriss DJ, Atkinson G. Ethical standards in sport and exercise science research: 2016 update. Int J Sports Med 2015; 36: 1121-1124

[9] Jeukendrup A, Van Diemen A. Heart rate monitoring during training and competition in cyclists. J Sports Sci 1998; 16: 1-9

[10] Kay AD, Blazevich AJ. Effect of acute static stretch on maximal muscle performance: A systematic review. Med Sci Sports Exerc 2012; 44: 154-164

[11] Kistler BM, Walsh MS, Horn TS, Cox RH. The acute effects of static stretching on the sprint performance of collegiate men in the 60 -and 100-m dash after a dynamic warm-up. J Strength Cond Res 2010; 24: 2280-2284

[12] Lakens D. Calculating and reporting effect sizes to facilitate cumulative sciences: a pratical primer for t-tests and ANOVAs. Front Psychol 2013; 4: 863

[13] Little T, Williams AG. Effects of differential stretching protocols during warm-ups on high-speed motor capacities in professional soccer players. J Strength Cond Res 2006; 20: 203-307

[14] Magnusson P, Renström P. The European College of Sports Sciences Position statement: The role of stretching exercises in sports. Eur J Sport Sci 2006; 6: 87-91

[15] McGowan C], Pyne DB, Thompson KG, Rattray B. Warm-up strategies for sport and exercise: Mechanisms and applications. Sports Med 2015; 45: 1523-1546

[16] McHugh MP, Cosgrave CH. To stretch or not to stretch: The role of stretching in injury prevention and performance. Scand J Med Sci Sports 2010; 20: 169-181 
[17] Mizuno T, Matsumoto M, Umemura Y. Viscoelasticity of the muscletendon unit is returned more rapidly than range of motion after stretching. Scand J Med Sci Sports 2013; 23: 23-30

[18] Mizuno T, Matsumoto M, Umemura Y. Stretching-induced deficit of maximal isometric torque is restored within 10 minutes. J Strength Cond Res 2014; 28: 147-153

[19] Morse Cl, Degens H, Seynnes OR, Maganaris CN, Jones DA. The acute effect of stretching on the passive stiffness of the human gastrocnemius muscle tendon unit. J Physiol 2008; 586: 97-106

[20] Needham RA, Morse Cl, Degens H. The acute effect of different warm-up protocols on anaerobic performance in elite youth soccer players. J Strength Cond Res 2009; 23: 2614-2620

[21] Neiva HP, Marques MC, Barbosa TM, Izquierdo M, Marinho DA. Warm-up and performance in competitive swimming. Sports Med 2014; 44: 319-330

[22] Neiva HP, Marques MC, Barbosa TM, Izquierdo M, Viana JL, Marinho DA. Effects of $10 \mathrm{~min}$ vs. 20 min passive rest after warm-up on $100 \mathrm{~m}$ freestyle time-trial performance: A randomized crossover study. J Sci Med Sport 2017; 20: 81-86

[23] Neiva HP, Marques MC, Barbosa TM, Izquierdo M, Viana JL, Teixeira AM, Marinho DA. Warm-up for sprint swimming: Race-pace or aerobic stimulation? A randomized study. ] Strength Cond Res 2016, [epub ahead of print] doi:10.1519/JSC.0000000000001701

[24] Ribeiro YS, Del Vecchio FB. Meta-analysis of the acute effects of stretching on high intensity sprint performance. Rev Bras Educ Fis Esporte 2011; 25: 567-581
[25] Rosenbaum D, Hennig EM. The influence of stretching and warm-up exercises on Achilles tendon reflex activity. J Sports Sci 1995; 13: 481-490

[26] Saez Saez De Villarreal E, González-Badillo JJ, Izquierdo M. Optimal warm-up stimuli of muscle activation to enhance short and long-term acute jumping performance. Eur J Appl Physiol 2007; 100: 393-401

[27] Turki O, Chaouachi A, Behm DG, Chtara H, Chtara M, Bishop D, Chamari K, Amri M. The effect of warm-ups incorporating different volumes of dynamic stretching on 10-and 20 - $\mathrm{m}$ sprint performance in highly trained male athletes. J Strength Cond Res 2012; 26: 63-72

[28] Winchester JB, Nelson AG, Landin D, Young MA, Schexnayder IC. Static stretching impairs sprint performance in collegiate track and field athletes. J Strength Cond Res 2008; 22: 13-19

[29] Witvrouw E, Mahieu N, Danneels L, McNair P. Stretching and injury prevention. Sports Med 2004; 34: 443-449

[30] Yaicharoen P, Wallman K, Morton A, Bishop D. The effect of warm-up on intermittent sprint performance and selected thermoregulatory parameters. J Sci Med Sport 2012; 15: 451-456

[31] Young WB, Behm DG. Effects of running, static stretching and practice jumps on explosive force production and jumping performance. J Sports Med Phys Fitness. 2003; 43: 21-27

[32] Young W, Elias G, Power J. Effects of static stretching volume and intensity on plantar flexor explosive force production and range of motion. J Sports Med Phys Fitness. 2006; 46: 403-411 